IJMMS 29:10 (2002) 579-583

PII. S0161171202007676

http://ijmms.hindawi.com

(c) Hindawi Publishing Corp.

\title{
DERIVATIONS IN BANACH ALGEBRAS
}

\section{KYOO-HONG PARK, YONG-SOO JUNG, and JAE-HYEONG BAE}

\author{
Received 23 October 2000 and in revised form 2 June 2001
}

\begin{abstract}
We present some conditions which imply that a derivation on a Banach algebra maps the algebra into its Jacobson radical.
\end{abstract}

2000 Mathematics Subject Classification: 47B47, 46H99.

1. Introduction. Throughout this paper, $A$ represents an associative algebra over the complex field $\mathbb{C}$, and the Jacobson radical of $A$ and the center of $A$ are denoted by $\operatorname{rad}(A)$ and $Z(A)$, respectively. Let $I$ be any closed (2-sided) ideal of the Banach algebra $A$. Then let $Q_{I}$ denote the canonical quotient map from $A$ onto $A / I$. Recall that an algebra $A$ is prime if $a A b=\{0\}$ implies that either $a=0$ or $b=0$. A mapping $f: A \rightarrow A$ is called commuting (resp., centralizing) if $[f(x), x]=0$ (resp., $[f(x), x] \in Z(A)$ ) for all $x \in A$. More generally, for a positive integer $n$, we define a mapping $f$ to be $n$ commuting (resp., $n$-centralizing) if $\left[f(x), x^{n}\right]=0$ (resp., $\left[f(x), x^{n}\right] \in Z(A)$ ) for all $x \in A$. A linear mapping $d: A \rightarrow A$ is called a derivation if $d(x y)=d(x) y+x d(y)$ for all $x, y \in A$.

The Singer-Wermer theorem, which is a classical theorem of Banach algebra theory, states that every continuous derivation on a commutative Banach algebra maps into its Jacobson radical [9], and Thomas [10] proved that the Singer-Wermer theorem remains true without assuming the continuity of the derivation. (This generalization is called the Singer-Wermer conjecture.) On the other hand, Posner [6] obtained two fundamental results in 1957: (i) the first result (the so-called Posner's first theorem) asserts that if $d$ and $g$ are derivations on a 2-torsion free prime ring such that the product $d g$ is also a derivation, then either $d=0$ or $g=0$. (ii) The second result (the so-called Posner's second theorem) states that if $d$ is a centralizing derivation on a noncommutative prime ring, then $d=0$. As an analytic analogue of Posner's second theorem, Mathieu and Runde [5, Theorem 1] generalized the Singer-Wermer conjecture by proving that every centralizing derivation on a Banach algebra maps into its Jacobson radical. The main objective of this paper is to obtain a generalization (Theorem 2.3) of the above Singer-Wermer conjecture which is inspired by Posner's first theorem.

2. Results. To prove our main result we need the following two lemmas.

LEMMA 2.1. Let $d$ and $g$ be derivations on a noncommutative prime algebra A. If there exist a positive integer $n$ and $\alpha \in \mathbb{C}$ such that $\alpha d^{2}+g$ is $n$-commuting on $A$, then both $d=0$ and $g=0$ on $A$. 
Proof. For the convenience, we write $f$ instead of $\alpha d^{2}+g$. Then the assumption of the lemma can be written in the form

$$
\left[f(x), x^{n}\right]=0
$$

for all $x \in A$. For $\alpha=0$, the result is obtained from [3, Corollary, page 3713]. Let $\alpha \neq 0$. Substituting $x+\lambda y(\lambda \in \mathbb{C})$ for $x$ in (2.1), we obtain

$$
\lambda Q_{1}(x, y)+\lambda^{2} Q_{2}(x, y)+\cdots+\lambda^{n} Q_{n}(x, y)=0, \quad x, y \in A,
$$

where $Q_{i}(x, y)$ denotes the sum of terms involving $i$ factors of $y$ in the expansion of $\left[f(x+\lambda y),(x+\lambda y)^{n}\right]=0$. Since $\lambda$ is arbitrary, we have

$$
\begin{aligned}
Q_{1}(x, y)= & {\left[f(y), x^{n}\right]+\left[f(x), x^{n-1} y\right] } \\
& +\left[f(x), x^{n-2} y x\right]+\cdots+\left[f(x), y x^{n-1}\right]=0, \quad x, y \in A .
\end{aligned}
$$

Substituting $x y$ for $y$ in (2.3), we get

$$
\begin{aligned}
0= & x\left[f(x), x^{n-1} y\right]+[f(x), x] x^{n-1} y \\
& +x\left[f(x), x^{n-2} y x\right]+[f(x), x] x^{n-2} y x \\
& +\cdots+x\left[f(x), y x^{n-1}\right]+[f(x), x] y x^{n-1} \\
& +f(x)\left[y, x^{n}\right]+2 \alpha\left[d(x) d(y), x^{n}\right]+x\left[f(y), x^{n}\right], \quad x, y \in A ;
\end{aligned}
$$

and left multiplying (2.3) by $x$ and subtracting the result from (2.4), we have

$$
\begin{aligned}
0= & {[f(x), x] x^{n-1} y+[f(x), x] x^{n-2} y x+\cdots+[f(x), x] y x^{n-1} } \\
& +f(x)\left[y, x^{n}\right]+2 \alpha\left[d(x) d(y), x^{n}\right], \quad x, y \in A .
\end{aligned}
$$

In (2.5), replace $y$ by $y x$ to obtain

$$
\begin{aligned}
0= & {[f(x), x] x^{n-1} y x+[f(x), x] x^{n-2} y x^{2} } \\
& +\cdots+[f(x), x] y x^{n}+f(x)\left[y, x^{n}\right] x \\
& +2 \alpha\left[d(x) d(y), x^{n}\right] x+2 \alpha\left[d(x) y d(x), x^{n}\right], \quad x, y \in A
\end{aligned}
$$

and multiply by $x$ on the right in (2.5) to obtain

$$
\begin{aligned}
0= & {[f(x), x] x^{n-1} y x+[f(x), x] x^{n-2} y x^{2}+\cdots+[f(x), x] y x^{n} } \\
& +f(x)\left[y, x^{n}\right] x+2 \alpha\left[d(x) d(y), x^{n}\right] x, \quad x, y \in A .
\end{aligned}
$$

We now subtract (2.7) from (2.6) to get

$$
d(x) y d(x) x^{n}-x^{n} d(x) y d(x)=0, \quad x, y \in A .
$$

Replacing $y$ by $y d(x) z$ in (2.8), we obtain

$$
d(x) y d(x) z d(x) x^{n}-x^{n} d(x) y d(x) z d(x)=0, \quad x, y, z \in A .
$$


According to (2.8), we can write, in relation (2.9), $x^{n} d(x) z d(x)$ for $d(x) z d(x) x^{n}$ and $d(x) y d(x) x^{n}$ instead of $x^{n} d(x) y d(x)$, which gives

$$
d(x) y\left[d(x), x^{n}\right] z d(x)=0, \quad x, y, z \in A .
$$

From (2.10) and primeness of $A$, it follows that, for any $x \in A$ we have either $\left[d(x), x^{n}\right]$ $=0$ or $d(x)=0$. In any case $\left[d(x), x^{n}\right]=0$ for all $x \in A$, which yields $d=0$ on $A$ by [3, Corollary, page 3713]. Now the initial hypothesis yields that $\left[g(x), x^{n}\right]=0, x \in A$, so $g=0$ on $A$, which completes the proof of the lemma.

LEMMA 2.2. Let $d$ be a derivation on a Banach algebra $A$ and $J$ a primitive ideal of $A$. If there exists a real constant $K>0$ such that $\left\|Q_{J} d^{n}\right\| \leq K^{n}$ for all $n \in \mathbb{N}$, then $d(J) \subseteq J$.

Proof. See [11, Lemma 1.2].

Now we prove our main result.

THEOREM 2.3. Let $d$ and $g$ be derivations on a Banach algebra A. If there exist a positive integer $n$ and $\alpha \in \mathbb{C}$ such that $\alpha d^{2}+g$ is $n$-commuting on $A$, then both $d$ and $g$ map A into $\operatorname{rad}(A)$.

Proof. Let $J$ be any primitive ideal of $A$. Using Zorn's lemma, we find a minimal prime ideal $P$ contained in $J$, and hence $d(P) \subseteq P$ and $g(P) \subseteq P$ (see [5, Lemma]). Suppose first that $P$ is closed. Then the derivations $d$ and $g$ on $A$ induce the derivations $\bar{d}$ and $\bar{g}$ on the Banach algebra $A / P$, defined by $\bar{d}(x+P)=d(x)+P$ and $\bar{g}(x+P)=$ $g(x)+P(x \in A)$. In case $A / P$ is commutative, both $\bar{d}(A / P)$ and $\bar{g}(A / P)$ are contained in the Jacobson radical of $A / P$ by [10]. We consider the case when $A / P$ is noncommutative. The assumption that $\alpha d^{2}+g$ is $n$-commuting on $A$ gives that the mapping $\alpha \bar{d}^{2}+\bar{g}$ is $n$-commuting on $A / P$. Since $A / P$ is a prime algebra, it follows from Lemma 2.1 that both $\bar{d}=0$ and $\bar{g}=0$ on $A / P$. Consequently, we see that both $d(A) \subseteq J$ and $g(A) \subseteq J$. If $P$ is not closed, then we see that $\mathscr{Y}(d) \subseteq P$ by [2, Lemma 2.3], where $\mathscr{S}(T)$ is the separating space of a linear operator $T$. Then we have, by [8, Lemma 1.3], $\mathscr{S}\left(Q_{\bar{P}} d\right)=\overline{Q_{\bar{P}}(\mathscr{S}(d))}=\{0\}$ whence $Q_{\bar{P}} d$ is continuous on $A$. This means that $Q_{\bar{P}} d(\bar{P})=\{0\}$, that is, $d(\bar{P}) \subseteq \bar{P}$. Hence, we see that $d$ induces a derivation $\tilde{d}$ on the Banach algebra $A / \bar{P}$, defined by $\tilde{d}(x+\bar{P})=d(x)+\bar{P}(x \in A)$. This shows that we can define a map

$$
\Psi \tilde{d}^{n} Q_{\bar{P}}: A \longrightarrow A / \bar{P} \longrightarrow A / \bar{P} \longrightarrow A / J
$$

by $\Psi \tilde{d}^{n} Q_{\bar{P}}(x)=Q_{J} d^{n}(x)(x \in A, n \in \mathbb{N})$, where $\Psi$ is the canonical induced map from $A / \bar{P}$ onto $A / J$ (the relation $\bar{P} \subseteq J$ guarantees its existence). The continuity of $\tilde{d}$ is clear from [8, Lemma 1.4], and hence yields that $\left\|Q_{J} d^{n}\right\| \leq\|\tilde{d}\|^{n}$ for all $n \in \mathbb{N}$. Now, according to Lemma 2.2, we obtain that $d(J) \subseteq J$. Following the same argument with $g$, we see that $g(J) \subseteq J$. Then the derivations $d$ and $g$ on $A$ induce the derivations $\hat{d}$ and $\hat{g}$ on the Banach algebra $A / J$, defined by $\hat{d}(x+J)=d(x)+J$ and $\hat{g}(x+J)=g(x)+J$ $(x \in A$ ). The rest follows as when $P$ is closed since the primitive algebra $A / J$ is prime. So we also obtain that $d(A) \subseteq J$ and $g(A) \subseteq J$. Since $J$ was arbitrary, we arrive at the conclusion that $d(A) \subseteq \operatorname{rad}(A)$ and $g(A) \subseteq \operatorname{rad}(A)$. 
A mapping $f: A \rightarrow A$ is said to be skew-centralizing if $\langle f(x), x\rangle \in Z(A)$ for all $x \in A$, where $\langle a, b\rangle$ denotes the Jordan product $a b+b a$.

COROLLARY 2.4. Let $d$ and $g$ be derivations on a Banach algebra A. If there exists $\alpha \in \mathbb{C}$ such that $\alpha d^{2}+g$ is skew-centralizing on $A$, then both $d$ and $g$ map $A$ into $\operatorname{rad}(A)$.

Proof. Since $\left\langle\alpha d^{2}(x)+g(x), x\right\rangle \in Z(A)$ for all $x \in A$, we obtain that $\left[\left\langle\alpha d^{2}(x)+\right.\right.$ $g(x), x\rangle, x]=0$ for all $x \in A$. From the relation

$$
\begin{aligned}
0 & =\left[\left\langle\alpha d^{2}(x)+g(x), x\right\rangle, x\right] \\
& =\left\langle\left[\alpha d^{2}(x)+g(x), x\right], x\right\rangle \\
& =\left[\alpha d^{2}(x)+g(x), x^{2}\right],
\end{aligned}
$$

we see that $\alpha d^{2}+g$ is 2-commuting, and hence Theorem 2.3 guarantees the conclusion.

As a noncommutative version of the Singer-Wermer theorem, we also obtain the next result by using Lemma 2.1.

THEOREM 2.5. Let $d$ and $g$ be continuous derivations on a Banach algebra A. If there exist a positive integer $n$ and $\alpha \in \mathbb{C}$ such that the mapping $\alpha d^{2}+g$ is $n$-centralizing on $A$, then both $d$ and $g$ map $A$ into $\operatorname{rad}(A)$.

Proof. Given any primitive ideal $J$ of $A$, we have $d(J) \subseteq J$ and $g(J) \subseteq J$ by [7, Theorem 2.2]. Thus we can suppose that $A$ is primitive. From $\left[\alpha d^{2}(x)+g(x), x^{n}\right] \in$ $Z(A)$ for all $x \in A$, we obtain $\left[\left[\alpha d^{2}(x)+g(x), x^{n}\right], x^{n}\right]=0$, and hence $\left[\alpha d^{2}(x)+\right.$ $g(x), x^{n}$ ] is quasinilpotent by the Kleinecke-Shirokov theorem [1, Proposition 18.13]. Since $Z(A)$ is trivial, it follows that $\left[\alpha d^{2}(x)+g(x), x^{n}\right]$ is a scalar multiple of 1 , and so $\left[\alpha d^{2}(x)+g(x), x^{n}\right]=0$ for all $x \in A$. Note that a commutative primitive Banach algebra is isomorphic to the complex field $\mathbb{C}$. Hence we also can assume that $A$ is noncommutative. Now, the primeness of $A$ and Lemma 2.1 allows that both $d=0$ and $g=0$ on $A$, which gives the result.

We do not know whether Theorem 2.5 can be proved without the continuity assumption. However, in the special case when the Banach algebra is semisimple, we obtain the following result.

COROLlARY 2.6. Let $d$ and $g$ be derivations on a semisimple Banach algebra A. If there exist a positive integer $n$ and $\alpha \in \mathbb{C}$ such that $\alpha d^{2}+g$ is $n$-centralizing on $A$, then both $d=0$ and $g=0$ on $A$.

Proof. The fact that every derivation on a semisimple Banach algebra is continuous [4, Remark 4.3] guarantees the conclusion.

\section{REFERENCES}

[1] F. F. Bonsall and J. Duncan, Complete Normed Algebras, Ergebnisse der Mathematik und ihrer Grenzgebiete, vol. 80, Springer-Verlag, New York, 1973.

[2] J. Cusack, Automatic continuity and topologically simple radical Banach algebras, J. London Math. Soc. (2) 16 (1977), no. 3, 493-500. 
[3] Q. Deng and H. E. Bell, On derivations and commutativity in semiprime rings, Comm. Algebra 23 (1995), no. 10, 3705-3713.

[4] B. E. Johnson and A. M. Sinclair, Continuity of derivations and a problem of Kaplansky, Amer. J. Math. 90 (1968), 1067-1073.

[5] M. Mathieu and V. Runde, Derivations mapping into the radical. II, Bull. London Math. Soc. 24 (1992), no. 5, 485-487.

[6] E. C. Posner, Derivations in prime rings, Proc. Amer. Math. Soc. 8 (1957), 1093-1100.

[7] A. M. Sinclair, Continuous derivations on Banach algebras, Proc. Amer. Math. Soc. 20 (1969), 166-170.

[8] _ Automatic Continuity of Linear Operators, London Mathematical Society Lecture Note Series, no. 21, Cambridge University Press, Cambridge, 1976.

[9] I. M. Singer and J. Wermer, Derivations on commutative normed algebras, Math. Ann. 129 (1955), 260-264.

[10] M. P. Thomas, The image of a derivation is contained in the radical, Ann. of Math. (2) 128 (1988), no. 3, 435-460.

[11] _ Primitive ideals and derivations on noncommutative Banach algebras, Pacific J. Math. 159 (1993), no. 1, 139-152.

Kyoo-Hong Park: Department of Mathematics Education, Seowon University, Chonguu, Chungbuk 361-742, Korea

E-mail address: parkkh@seowon.ac.kr

Yong-Soo Jung: Department of Mathematics, Chungnam National University, TAEJON 305-764, KOREA

E-mail address: ysjung@math.cnu.ac.kr

Jae-Hyeong Bae: Department of Mathematics, Chungnam national University, TAEJON 305-764, KOREA

E-mail address: jhbae@math.cnu.ac.kr 


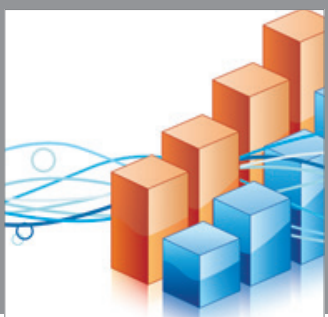

Advances in

Operations Research

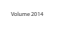

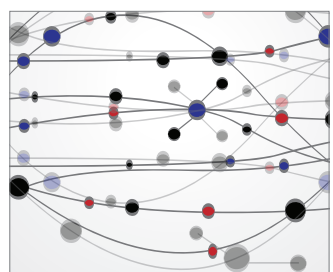

\section{The Scientific} World Journal
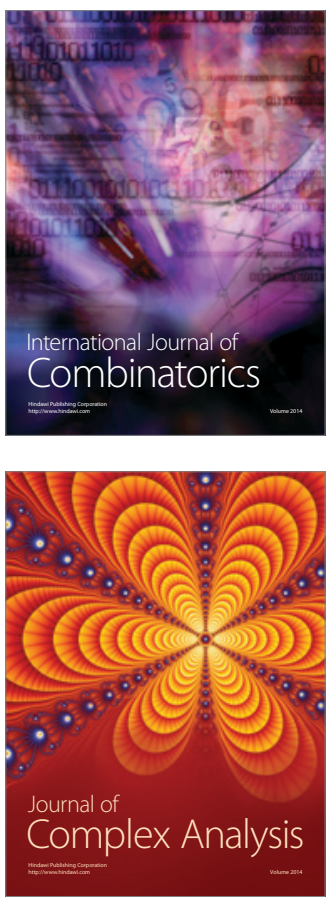

International Journal of

Mathematics and

Mathematical

Sciences
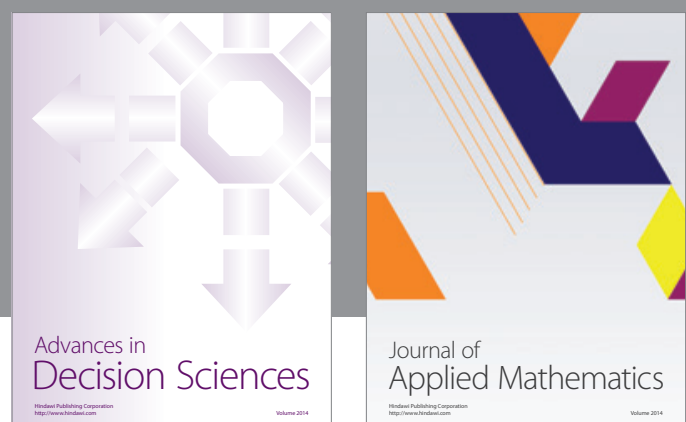

Journal of

Applied Mathematics
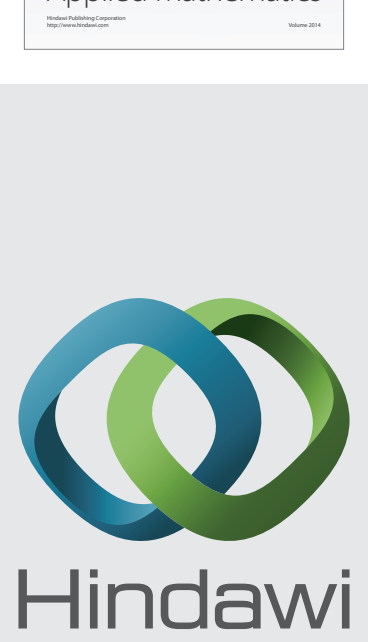

Submit your manuscripts at http://www.hindawi.com
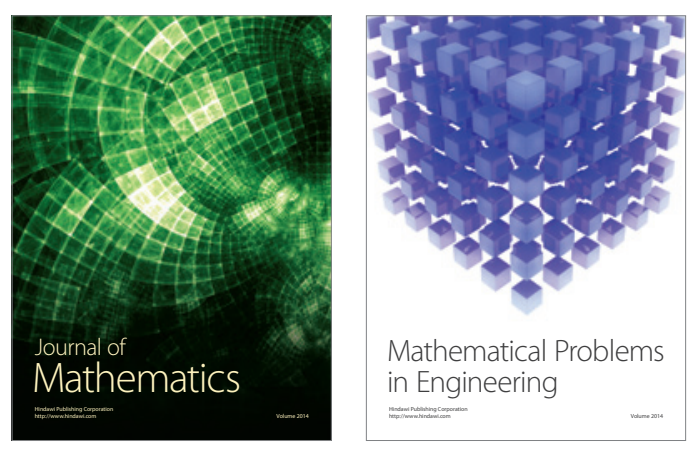

Mathematical Problems in Engineering
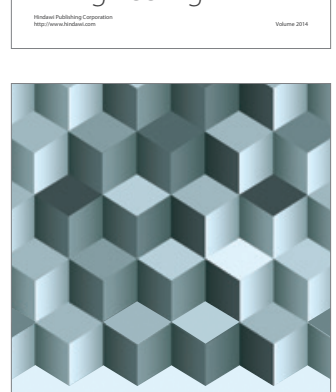

Journal of

Function Spaces
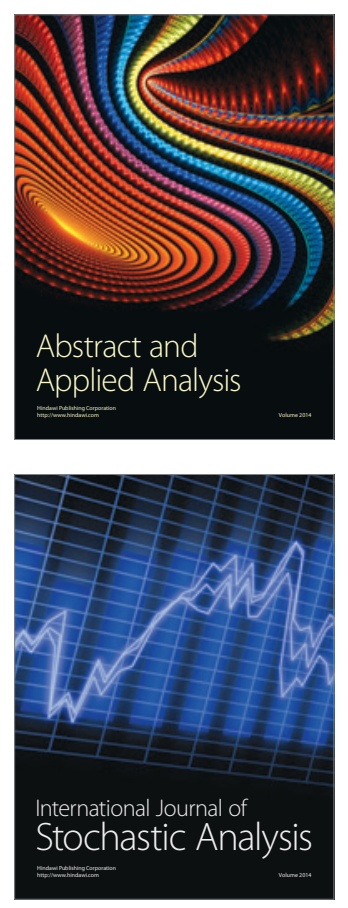

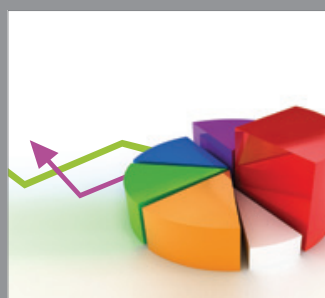

ournal of

Probability and Statistics

Promensencen
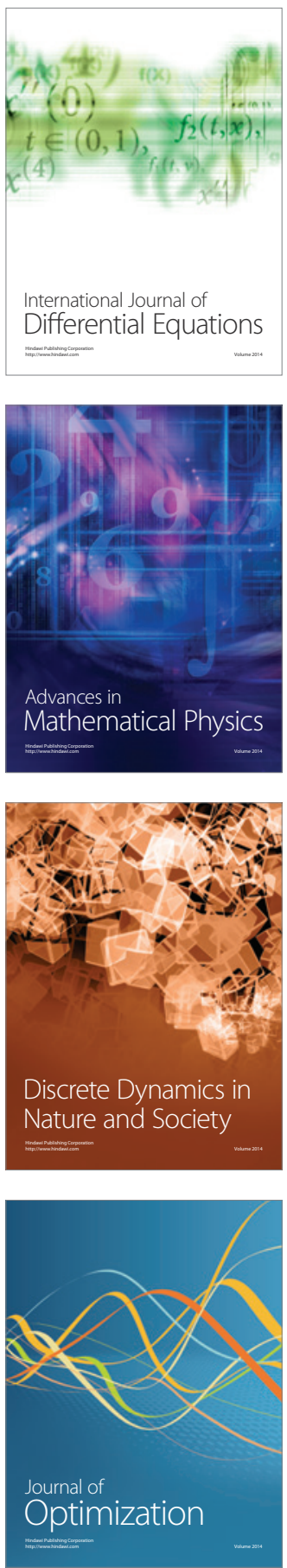